\title{
NOUVELLE
}

\section{nAG sur la piste de la médecine régénérative}

Sophie Vriz
Université Paris 7-Denis Diderot, Inserm U770, Université Paris XI, 80, rue du Général Leclerc, 94276 Le Kremlin-Bicêtre, France. vriz@univ-paris-diderot.fr
> La capacité de régénérer des parties lésées ou amputées varie beaucoup d'une espèce à l'autre. Chez les vertébrés, seuls les poissons téléostéens et les amphibiens urodèles conservent naturellement cette possibilité tout au long de la vie [1]. Aussi, la compréhension des mécanismes de régénération de structures complexes chez la salamandre adulte peut-elle ouvrir des pistes pour la recherche en médecine régénérative chez l'homme. Régulièrement pendant ces 20 dernières années, les travaux de Jeremy Brockes ont bousculé les idées reçues et permis l'émergence de nouveaux concepts en médecine régénérative [2-4]. Le travail de cette équipe, publié par la revue Science en novembre 2007, décrit le rôle d'une petite molécule sécrétée dans la régénération des pattes chez l'adulte [5]; pour la première fois, il donne un éclairage nouveau sur l'interdépendance des trois éléments essentiels à l'initiation de la régénération: la formation d'un épiderme de cicatrisation, un défaut d'identité de position entre cet épiderme et le tissu sous-jacent, la présence de nerfs vivants dans le moignon.

\section{Le blastème de régénération}

La régénération épimorphique implique un renouvellement coordonné de nombreux types cellulaires à partir d'un petit groupe de cellules. Sa transposition en médecine régénérative nécessite de comprendre les voies de signalisation impliquées d'une part dans la plasticité in vivo des cellules différenciées adultes, et d'autre part dans le maintien d'une identité de position au niveau de ces cellules, élément essentiel pour que la reconstruction de la structure se fasse en harmonie avec ce qui l'entoure
$[6,7]$. Chez l'amphibien, la régénération se fait par dédifférenciation de cellules (fibroblastes, cellules musculaires, ostéoblastes, cellules de Schwann, etc.) en cellules souches $[3,8]$. Ces cellules souches migrent au niveau du plan d'amputation et prolifèrent pour former un amas de cellules pluripotentes -appelé blastème de régénération- à partir duquel se reformera la partie manquante [1]. Au cours de ce processus, l'épiderme de cicatrisation produit un ou des ligands de la voie Fgf (fibroblast growth factor) indispensable à la formation du blastème; ainsi, chez le poisson zèbre l'inhibition de la voie Fgf suffit à bloquer la formation du blastème [1]. Si la voie Fgf est impliquée dans de nombreux dialogues entre épiderme et mésenchyme, ses cibles dans le processus de régénération sont encore mal connues. Des observations anciennes ont montré qu'une section des fibres nerveuses au niveau de la moelle épinière bloque également la formation du blastème [2]. Chez tous les vertébrés, l'innervation est indispensable à la régénération. Les fibres nerveuses coupées régénèrent en même temps que la patte et cette repousse est indispensable à la régénération du membre [9]. Enfin, le blastème reconstitue une structure correspondant précisément à la partie distale manquante, quel que soit le niveau d'amputation. L'information de position intervient dans la détection de disparités entre cellules voisines, et contribue à induire les remaniements cellulaires à l'origine du processus de régénération. Jeremy Brockes a montré, il y a quelques années, que l'identité de position des cellules souches est donnée par la protéine Prod-1/ CD59, exprimée sous forme d'un gradient proximo-distal dans la patte adulte [4] (Figure 1). Prod-1 est une glycoprotéine fixée à la membrane par une ancre GPI ( $g l y$ cosyl-phosphatidyl-inositol). En recherchant des ligands potentiels de Prod-1, l'équipe de Jeremy Brockes a découvert le facteur responsable de la nécessité de l'innervation et de l'interdépendance de l'épithélium de cicatrisation et des fibres nerveuses dans la régénération: nAG (newt anterior gradient 2 protein) [5].

\section{nAG, un facteur clé}

pour comprendre le lien

entre innervation et régénération

Après amputation, les axones lésés se rétractent dans le moignon avant de repousser dans la gaine de cellules de Schwann

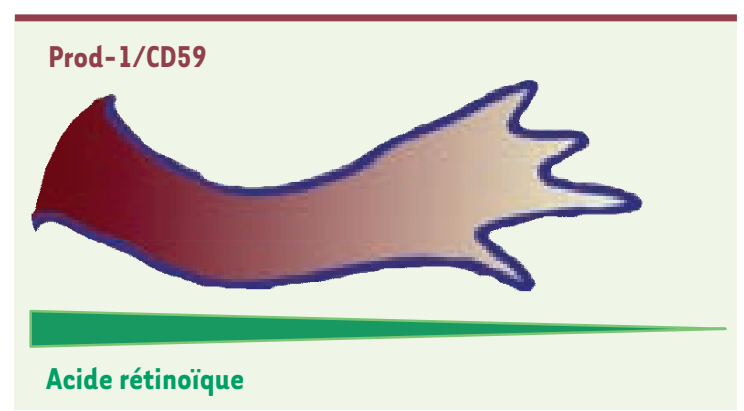

Figure 1. Gradient d'expression de la protéine Prod1/CD59 dans la patte adulte. La protéine Prodl est l'orthologue de la protéine humaine CD59, d'abord identifiée comme régulateur de la voie du complément. La distribution de cette protéine membranaire suivant l'axe proximo-distal de la patte adulte est modulée par l'acide rétinoïque et directement impliquée dans l'information de position au cours de la régénération [4]. 
(Figure 2). Deux vagues d'expression de nAG sont observées; dans un premier temps dans les cellules de Schwann au niveau desquelles l'axone s'est rétracté (Figure $2 A)$, puis dans l'épithélium de cicatrisation (Figure 2B). La protéine $\mathrm{nAG}$ stimule la prolifération de cellules en culture [5] et son expression est régulée par la voie Fgf chez le xénope, ce qui en fait une cible potentielle des ligands de la voie Fgf exprimés dans l'épithélium de cicatrisation. Aucune expression de nAG n'est détectée si les axones sont éliminés avant amputation (Figure $2 C$ ), ce qui montre que cette expression est dépendante de la présence d'un nerf vivant. L'équipe de Jeremy Brockes a induit une expression ubiquitaire de $n A G$ dans les cellules du moignon d'une patte dénervée par électroporation d'un plasmide codant nAG (Figure 2 $\varepsilon$ ). Cette faible expression ubiquitaire est suffisante pour induire la deuxième vague d'expression de nAG dans l'épithélium de cicatrisation (Figure 2F) et permettre une régénération complète des pattes dénervées (Figure 2G). Ce résultat donne la clé pour comprendre pourquoi la régénération dépend de l'innervation, et rend compte, au niveau moléculaire, du lien entre information de position et prolifération cellulaire. De plus, il donne une explication mécanistique aux travaux de biologie expérimentale portant sur l'identité de

\section{Expression de nAG, un ligand de Prodl}

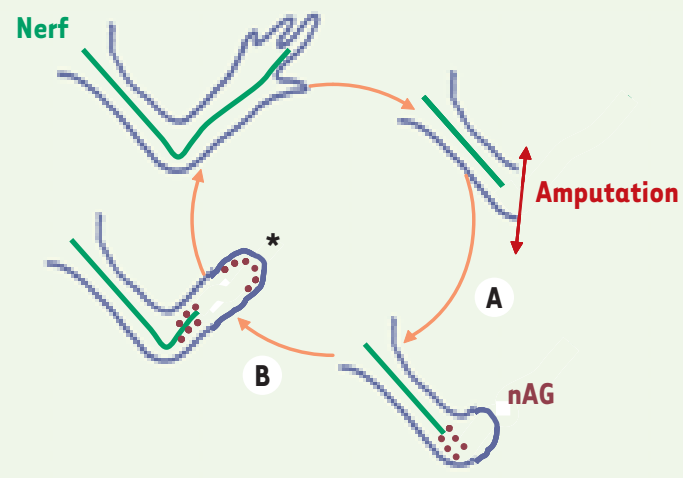

\section{nAG restaure la régénération de pattes dénervées}

Section du nerf

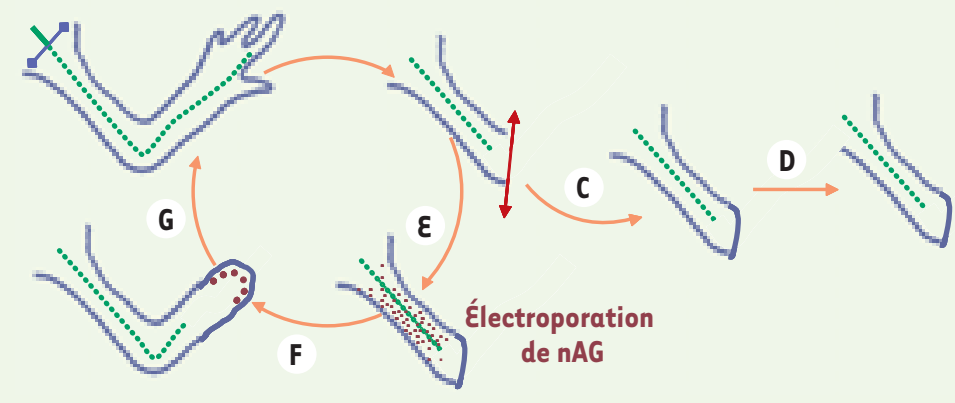

Figure 2. nAG : clé de la dépendance vis-à-vis des nerfs pour la régénération des pattes chez l'adulte. Expression de nAG, un ligand de Prodl. Identifiée sur la base de sa fixation à Prodl, nAG est une petite protéine sécrétée $(27 \mathrm{kDa})$, dont l'expression est induite par l'amputation (A). Elle est d'abord détectée dans les cellules de Schwann de l'extrémité du moignon puis dans des groupes de cellules de l'épiderme de cicatrisation* $(B)$. $n A G$ restaure la régénération de pattes dénervées. Aucune expression de $\mathrm{nAG}$ n'est détectée après amputation de pattes dénervées au préalable (C). Celles- ci sont incapables de former un blastème et de régénérer ( $D$ ). L'introduction d'ADN exprimant la protéine $n A G$ dans les cellules du moignon dépourvu d'axone $(\varepsilon)$, induit l'expression de $\mathrm{nAG}$ dans l'épithélium de cicatrisation (F) et restaure la régénération de la patte (G).

position, au cours de la régénération, aussi bien chez l'insecte que chez l'amphibien [10]. Ces résultats ouvrent aussi de nombreux champs d'investigations en particulier en ce qui concerne le rôle des fibres nerveuses au cours du développement du membre et de sa régénération chez l'adulte. En effet si Yntema a montré dans les années 1950 que les pattes peuvent se développer et régénérer sans nerfs, Charles et Mary Thornton ont montré que la repousse du nerf lésé dans le nouveau tissu était essentielle pour la dépendance de la régénération vis-à-vis du nerf [9]. Dans ce contexte, la découverte de $\mathrm{nAG}$ apporte un nouvel outil pour étudier ce phénomène.

Jeremy Brockes et ses collaborateurs montrent donc sans ambigüité qu'à elle seule, $\mathrm{nAG}$ induit la reformation d'une patte complète dans un contexte où la formation du blastème est inhibée in vivo. Jeremy Brockes avait déjà souligné qu'une des limitations à la régénération de structures complexes chez les mammifères était l'absence de blastème [6]. La protéine $n A G$ pourrait être le promoteur de régénération qui nous manque. $\diamond$

nAG, a molecular clue

for nerve dependence of regeneration

\section{RÉFÉRENCES}

1. Tawk M, Vriz S. La régénération des appendices chez les vertébrés : un modèle expérimental ancien pour étudier les cellules souches chez l'adulte. Med Sci (Paris) $2003 ; 19: 465-71$.

2. Brockes JP. Mitogenic growth factors and nerve dependance of limb regeneration. Science 1984 ; 225: 1280-7.

3. Lo DC, Allen F, Brockes JP. Reversal of muscle differentiation during urodele limb regeneration. Proc Natl Acad Sci USA 1993; $90: 7230-4$

4. Da Silva SM, Gates PB, Brockes JP. The newt ortholog of CD59 is implicated in proximodistal identity during amphibian limb regeneration. Dev Cell 2002 ; 3: $547-55$.

5. Kumar A, Godwin, JW, Gates, PB et al. Molecular basis for the nerve dependence of limb regeneration in an adult vertebrate. Science 2007 ; 318 : $772-7$.

6. Brockes JP, Kumar A. Appendage regeneration in adult vertebrates and implications for regenerative medicine. Science 2005 ; 310 : 1919-23.

7. Stocum DL. Amphibian regeneration and stem cells. Curr Top Microbiol Immunol 2004 ; 280 : 1-70.

8. Echeverri K, Tanaka EM. Ectoderm to mesoderm lineage switching during axolotl tail regeneration. Science 2002; 298: 1993-6.

9. Brockes JP. The nerve dependence of amphibian limb regeneration. J Exp Biol 1987 ; 132 : 79-91.

10. French V, Bryant PJ, Bryant SV. Pattern regulation in epimorphic fields. Science $1976 ; 193:$ 969-81. 